\title{
Aktuelle Regionalisierungsprozesse in der Region Rhein-Main
}

\section{Current Regionalisation Processes in the Rhine-Main Region}

\begin{abstract}
Kurzfassung
In den Regionen Stuttgart und Hannover wurden bereits tiefgreifende Regionalreformen durchgeführt, die sich bewährt haben. Für das Rhein-Main-Gebiet wurde mit dem Ballungsraumgesetz hingegen eine Regionalstruktur eingeführt, die seit ihrer Einführung 2001 weniger erfolgreich war. Statt dessen ist die Debatte darum, wie die Region RheinMain zu strukturieren ist und welche Art der Steuerung ein solcher polyzentrischer Verdichtungsraum braucht, aufs Neue entbrannt. Der vorliegende Aufsatz versucht, aktuelle Regionalisierungsprozesse - ausgehend vom theoretischen Ansatz der handlungstheoretischen Sozialgeographie - nachzuzeichnen und Akteurskonstellationen zu identifizieren, die derzeit damit befasst sind, Vorschläge für eine zukunfts- und handlungsfähige Regionalstruktur im Rhein-Main-Gebiet zu schaffen bzw. in der entsprechenden Debatte Einfluss geltend zu machen. Dazu werden verschiedene Beispiele regionaler Initiativen und Organisationen vorgestellt und kritisch in ihrer Bedeutung und Wirksamkeit hinterfragt.
\end{abstract}

\begin{abstract}
Far-reaching regional reforms have already been carried out in the Stuttgart and Hanover regions that have proved to be effective. For the Rhine-Main area, on the other hand, a regional structure has been introduced with the Conurbation Act that has been less successful since its introduction in 2001. Instead the debate on how the Rhine-Main region should be structured and which kind of regulation such a polycentric urban agglomeration needs has broken out again. This article attempts to outline current regionalisation processes taking the theoretical approach of action-theoretical social geography as a point of departure and to identify constellations of actors who are currently occupied with developing suggestions for a future- and policy-oriented regional structure in the Rhine-Main area or with asserting their influence in the corresponding debate. In order to do this, different examples of regional initiatives and organisations are presented and questioned critically in terms of their significance and effectiveness.
\end{abstract}

\section{Was heißt „Regionalisierung“?}

Verschiedentlich wird diskutiert, dass die in Deutschland bestehenden Verwaltungsstrukturen mit ihren zahlreichen Ebenen in Verdichtungsräumen nicht mehr in der Lage seien, Lösungen für aktuelle Probleme zu finden. Die logische Konsequenz ist, für solche Räume eine Regionalreform zu fordern. Entsprechende Reformen wurden in den Regionen Stuttgart (1994) und Hannover (2001) durchgeführt. In der Region Rhein-Main hingegen wurde bis 1999 sehr heftig um eine Regionalreform gerungen. Den Stand der Debatte bis zu diesem Zeitpunkt hat Scheller (1998) beschrieben. Mit der Landtagswahl 1999 wurde die Regierung von SPD und Grünen durch eine CDU/FDP-Regierung unter Ministerpräsident $\mathrm{KOCH}$ abgelöst. In der Koali- 
tionsvereinbarung von CDU und FDP vom März 1999 wurde eine neue Regionalstruktur für das Rhein-MainGebiet angekündigt. Dieser Aufsatz wird sich mit den Entwicklungen in der Region seit 1999 befassen und die verschiedenen Regionalisierungsprozesse darlegen. Dazu sind zunächst einige Ausführungen zum Begriff „Regionalisierung“ notwendig.

In der Geographie werden Abgrenzungen auf der Basis funktionaler Kriterien meist als „Regionalisierungen“ bezeichnet. Für die Region Rhein-Main wurde eine solche Abgrenzung erstmals 1961 von Krenzlin (1961, S. 312-313) vorgelegt. Krenzlin vollzog damit für das Rhein-Main-Gebiet die Abkehr von der zuvor gebräuchlichen Methode der Regionalisierung nach "Landschaften“. Auch heute werden Regionalisierungen oft nach funktionalen Kriterien vollzogen: Die Abgrenzung der Region für den „Regionalatlas RheinMain" basiert bspw. auf Pendlerdaten und bedient sich zur Regionalisierung der Landkreisgrenzen (Rheinmainische Forschung 2000), auch wenn Wolf bereits 1990 (27) einräumte, dass „die Verwaltungs- und Planungsgrenzen ... den Raum eher (zerschneiden), als dass sie ihn als gemeinsamen Struktur- und Funktionalraum auffassen." Einen Schritt weiter geht Schamp (2001, S. 169), wenn er Regionen als Umschlagplätze für Informationen, Innovationen und Kapitalflüsse bezeichnet, die "hubs“ im "space of flows" seien und jenseits des klassischen Raumverständnisses anzusiedeln wären. Hinsichtlich der Funktion und Bedeutung der Rhein-Main-Region gelingt es Schamp zu belegen, dass diese hinsichtlich ihrer wirtschaftlichen Spezialisierung ein "hub“ in Europa ist. Dennoch ist es nicht möglich, solche Funktionen ohne konkrete räumliche Bezüge nachzuvollziehen (schon allein, weil statistische Daten nur für bestimmte administrative Raumeinheiten erhoben werden). Insofern wundert es nicht weiter, wenn Schamp zwar die ortsungebundene Funktion der Region innerhalb des europäischen Metropolensystems betont, $\mathbf{z u}$ Belegen aber auf eine "klassische Regionalisierung“ - das „IHKForum Rhein-Main“ - zurückgreift.

Nach Bahrenberg (2002, S. 53) entsprechen die genannten Regionalisierungen des Rhein-Main-Gebietes einem Verständnis von Geographie, das „auf den Nachweis der Existenz von Regionen und ... auf die Bestimmung ihrer territorialen Grenzen [gerichtet war], was häufig zu Empfehlungen an das politisch-administrative System führte, seine Territorialstruktur an die von der Geographie bestimmten Grenzen anzupassen“. Das Interesse der Geographie an den (politischen) Prozessen, die zu einer tatsächlichen Regionalisierung führen bzw. geführt haben, sei nach Bahrenberg nur zweitrangig gewesen. Bahrenberg stellt daher fest:
"Ohne Beteiligung der Politik keine Regionalisierung“ (Bahrenberg 2002, S. 61) und nennt die Politikberatung als wichtige Anwendung geographischer Arbeitsweisen. Daraus leitet sich die Frage ab, wie Politik regionalisiert oder anders formuliert wie wissenschaftliche Regionalisierungen vorgenommen und kommuniziert werden müssten, um von der Politik wahrgenommen und umgesetzt zu werden. Konkret ist nur ein „Fall“ bekannt, in dem wissenschaftliche Gutachten unmittelbar zu einer Neuordnung administrativer Strukturen geführt haben: Dies ist die bayrische Raumplanung, die auf einem Gutachten von Ruppert basiert, das ,eine entscheidende Grundlage für die Gliederung Bayerns in Planungsregionen [bildete]" (Ruppert/Paesler 1983, S. III). Seither haben sich geographische Regionalisierungen außerhalb politischer Regionalisierungsprozesse bewegt bzw. wurde nur versucht, sie zu typisieren (vgl. Scheller 1998, 1999) - es müssen also innerhalb der Politik andere Kräfte sein, die zu administrativen Regionalisierungen führen.

Im Folgenden sollen aktuelle Regionalisierungsprozesse aus der Region Rhein-Main vorgestellt werden. Bahrenberg rückt solche Prozesse in die Nähe der Systemtheorie und betont dabei die wechselseitige $\mathrm{Ab}$ hängigkeit von Wissenschaft und Politik (Bahrenberg 2002, S. 55). Besondere Bedeutung erlangen in diesem Zusammenhang Organisationen innerhalb der Gesellschaft. Der institutionelle Überbau bildet jedoch nur den Rahmen, in dem sich politische wie wissenschaftliche Akteure bewegen und unterschiedliche Regionalisierungen vornehmen. Daher ist es sinnvoller, Regionalisierungsprozesse aus einer akteurszentrierten Sichtweise zu betrachten. Erfahrungen mit Regionalisierungen in anderen Regionen zeigen, dass ihr Erfolg eher vom Wollen oder Nicht-Wollen politischer Akteure abhängt als von institutionellen Strukturen.

Damit rücken die Regionalisierungen in die Nähe der handlungstheoretischen Sozialgeographie (Werlen 2001a), in der die Analyse von Handlungsweisen eine große Rolle spielt. Diese werden von Rahmenbedingungen bestimmt, zu denen Gesetze und Normen ebenso gehören wie Artefakte. Dieser Rahmen ist es, der Handlungsmöglichkeiten eröffnet, einschränkt oder versagt. „Raum" wird so zu einem „Kürzel“, das sich auf die physisch-materielle Komponente der Handlungsverwirklichung bezieht (Werlen 2001a, S. 327-328). Dies gilt auch für die „Region“, die - handlungstheoretisch interpretiert - den Handlungsraum von Akteuren bezeichnet. Es ist somit davon auszugehen, dass eine von einem Akteur vorgenommene Regionalisierung stets mit dem Ziel vorgenommen wird, sich selbst einen bestimmten Handlungsspielraum zu eröffnen bzw. den Handlungsspielraum anderer einzu- 
schränken. Dass dazu keine konkrete räumliche $\mathrm{Ab}$ grenzung notwendig ist, die sich wiederum auf physisch-materielle Artefakte bezieht, unterstreicht noch einmal die Tatsache, dass - insbesondere in der Politik - seit Jahrzehnten um die Region Rhein-Main gerungen wird, obwohl noch immer zahlreiche Abgrenzungen nebeneinander stehen. Diese als „politische Regionalisierungen" zu bezeichnenden Abgrenzungen sind der Versuch, über ihre Territorialisierung ein Mittel der Machtausübung bzw. -kontrolle zu schaffen (Werlen 2001b, S. 41). Da - so Werlen - ebenfalls zu untersuchen ist, welche Konsequenzen diese Geographien in lokaler und globaler Hinsicht haben (Werlen 2001a, S. 311), rücken die derzeit in der Region RheinMain diskutierten Vorschläge zu einer neuen Regionalstruktur in den Mittelpunkt der handlungszentrierten Sozialgeographie, da es darum geht, eine Regionalstruktur zu schaffen, die den Akteuren (aus der Sicht der Region selbst heraus betrachtet) ausreichende Handlungsmöglichkeiten eröffnet, so dass sich die Region künftig in der globalisierten Welt behaupten kann.

\section{Aktuelle Regionalisierungsprozesse}

\subsection{Ausgangslage}

Um aktuelle Regionalisierungen im Rhein-Main-Gebiet nachvollziehen zu können, ist es notwendig, darzulegen, warum eine neue Regionalstruktur sinnvoll ist. Zudem muss ein Blick auf die 2001 durch die Landesregierung geschaffene Regionalstruktur geworfen werden.

Die Region Rhein-Main zählt zu den wirtschaftsstärksten Regionen Deutschlands, wenn nicht Europas. Neuere Daten zeigen jedoch, dass das Rhein-Main-Gebiet im Vergleich zu anderen Regionen Europas zurückfällt (Wolf 2003, S. 16). Zudem ist zu beobachten, dass der Finanzplatz Frankfurt im Vergleich zu London an Bedeutung verloren hat (Schultheis 2003, S. 84). Hinzu kommen intraregionale Probleme, zu denen bspw. groBe Unterschiede in der kommunalen Finanzausstattung zählen, sodass die Diskussion über einen regionalen Finanzausgleich wiederbelebt wurde (Schultheis 2002). Ein im Auftrag der Landesregierung erstelltes Gutachten zum Finanzplatz Frankfurt kommt zu dem Schluss, dass die Region dringend der Stärkung bedarf - z.B. durch den Umzug der Bundesanstalt für Finanzdienstleistungsaufsicht (BAFin), Novellierung einschlägiger Gesetze, aber auch durch die Stärkung weicher Standortfaktoren in Stadt und Region -, um weiter zukunftsfähig zu bleiben (Spahn/van den Busch 2002).

\subsection{Das Ballungsraumgesetz}

Die hessische Landesregierung hat mit dem „Gesetz zur Stärkung der kommunalen Zusammenarbeit und Planung in der Region Rhein-Main vom 19.12.2000“ versucht, einen Vorschlag zur Lösung der regionalen Probleme zu unterbreiten. Dieses Gesetz löste den Umlandverband Frankfurt auf, schuf einen neuen Planungsverband sowie den „Rat der Region“ und ordnete an, dass innerhalb einer Übergangsfrist für regional bedeutsame Aufgaben freiwillige Kooperationsformen gefunden werden sollten. Wesentliche Inhalte wurden von einer Arbeitsgruppe formuliert, die eng mit Ministerpräsident Koch zusammengearbeitet hat.

Der Planungsverband bedeutet in diesem Zusammenhang eine klassische Regionalisierung, bei der administrative Einheiten zu einem neuen Planungsraum zusammengefasst wurden. Im Gegensatz zum Umlandverband, dessen Verbandsgebiet 43 Kommunen umfasste, sind es beim Planungsverband 75, für die erstmalig in Deutschland - ein Regionaler Flächennutzungsplan (RFNP) erstellt werden soll. Der Regionalisierung lagen keine funktionalen Kriterien zu Grunde, sondern sie ,erfolgte ... offenkundig im Bestreben, den Planungshorizont des politisch gewollten Regionalen Flächennutzungsplans zu beschränken [und] um die Arbeitsfähigkeit der Gemeindekammer zu gewährleisten" (Wolf/Scheller 2001, S. 11). Die Gemeindekammer, in die die Mitgliedskommunen Vertreter entsenden, legitimiert die Verbandsentscheidungen, wobei ein direkt gewähltes Kontrollorgan fehlt.

Der „Rat der Region“ ist für das Gebiet des Planungsverbandes zuständig und soll die Kooperation der Kommunen fördern, Regionalkonferenzen einberufen und festlegen, nach welchen Grundsätzen gemeinsam wahrzunehmende Aufgaben in der Region gelöst werden sollen.

Während der Diskussion des Gesetzes berücksichtigte die Landesregierung keine Änderungsvorschläge, sondern verwies darauf, dass Änderungen des vorgelegten Konzeptes nicht erfolgen würden, da die Einwände zu unterschiedlich seien und keine echten Alternativen vorgelegt worden seien, so der hessische Innenminister Bouffier (FR 24.08.2000). Als das Ballungsraumgesetz im Dezember 2000 verabschiedet wurde, deutete alles auf eine konservative Mehrheit in der Gemeindekammer hin. Einige Kritiker gingen sogar so weit zu vermuten, dass Gemeinden mit einem rot-grünen Magistrat bewusst nicht mit in den Ballungsraum aufgenommen wurden, um den Regierungsparteien eine Mehrheit in diesem Verband zu sichern und Einfluss auf dessen Arbeit nehmen zu können, da der vorgesehene Verbandsdirektor ebenfalls aus dem Umfeld des 
Ministerpräsidenten kommen sollte (FR 17.08.2000). Durch den Wechsel der politischen Mehrheit in der Gemeindekammer nach der Kommunalwahl 2001 wurde jedoch eine rot-grüne Verbandsspitze gewählt.

Weitere Regionalisierungen sollen für zahlreiche Einzelbereiche vorgenommen werden - z.B. Abfallentsorgung, Wasserver- und Abwasserentsorgung, überörtliche Freizeiteinrichtungen, Standortmarketing oder Regionalpark Rhein-Main. In diesen Bereichen sollen freiwillige Kooperationen z.B. in Form von Zweckverbänden gebildet werden. Da diese Aufgaben bislang von den Gebietskörperschaften wahrgenommen werden, ist zu erwarten, dass sich entsprechend „klassische" Regionalisierungen ergeben werden, z.B. als „Trinkwasserregion Rhein-Main“. Dem Gesetz zu Folge soll der Ballungsraum durch freiwillige Kooperationen jeweils insgesamt abgedeckt werden. Mit diesen flexiblen Regionalisierungen soll der Tatsache Rechnung getragen werden, dass für die verschiedenen Aufgaben unterschiedliche Abgrenzungen sinnvoll sind. Bspw. ist es bei der Trinkwasserversorgung von Frankfurt und seinem Umland logisch, den Vogelsberg und das Hessische Ried als Trinkwasserlieferanten ebenso mit in eine regionale Trägerschaft zu integrieren.

Insgesamt stellt das Ballungsraumgesetz eine Kombination einer "klassischen" territorialen Planungsregion (Verbandsgebiet des Planungsverbandes) und dynamischer aufgabenbezogener Regionalisierungen dar. Wiechmann (2000) merkt zur Kombination solch unterschiedlicher Regionen an, dass diese einem geänderten Verständnis von „Region“ geschuldet seien insbesondere im Zusammenhang mit akteurs- und netzwerkbasierten Regionalisierungen - und eine mögliche Lösung für künftige Regionalisierungen aus der Sicht der Raumplanung bilden könnten. Allerdings weist Wiechmann (2000, S. 182) auch darauf hin, dass solche "Parallelregionen" in der Praxis einer Bewährungsprobe harren - insofern kommt dem Ballungsraumgesetz nicht nur in Hinblick auf den RFNP der Charakter eines Pilotprojekts zu.

Für die freiwilligen Kooperationen unter der Leitung des „Rates der Region" wurde von der Landesregierung eine Übergangsfrist bis zum 31.12.2002 gewährt. Aber „der ... Rat der Region, der Grundsätze für diese interkommunale Zusammenarbeit aufstellen soll, war in den zwei Jahren seit Verabschiedung des Ballungsraumgesetzes nicht in der Lage, für die Organisation dieser Aufgaben tragfähige Vorschläge zu entwickeln“ (PVFRM 23.04.2003). Dies änderte sich auch 2003 nicht. So beklagte sich die FDP des Hochtaunuskreises in einer Pressemitteilung vom 14.03.2003: „Die Frankfurter Oberbürgermeisterin Petra Roth hatte in Absprache mit den Gebietskörperschaften bereits die zweite
Sitzung des Rats der Region in diesem Jahr mit dem Hinweis abgesagt, es gebe keine Tagesordnungspunkte. Die Vielzahl der Themen von Olympia über die Bewerbung als Kulturhauptstadt Europas, den Regionalpark bis zur völligen Neugestaltung der Region ist für Ruppert fast unüberschaubar" (Ruppert ist Vorsitzender der FDP Hochtaunus, Anm. d. Verf.).

So ist es bislang bei jenen Kooperationen geblieben, die bereits vor dem Ballungsraumgesetz Bestand hatten. Der Grundgedanke, nach dem flexibel neue zweckorientierte Regionalisierungen entstehen sollten, hat sich als nicht praktikabel erwiesen. Die „alten“ Kooperationen hingegen bilden vielfach nicht einmal den Ballungsraum ab. Ein typisches Beispiel ist die „Rhein-Main Abfall GmbH“ (RMA) bei der Abfallentsorgung, deren Zuständigkeitsbereich noch den ehemaligen Umlandverband nachzeichnet.

Auch für den "Regionalpark Rhein-Main “ ${ }^{1}$, zuvor in der Trägerschaft des Umlandverbandes Frankfurt, wurde erst nach Ablauf der Übergangsfrist eine Lösung gefunden, die als „regional“ bezeichnet werden kann und dies, obwohl der Regionalpark stets als regionales „Leuchtturmprojekt“ galt. So wurde erst im Juli 2003 eine Dachgesellschaft gegründet, der jedoch weder alle Landkreise, noch alle Kommunen der Region beitraten (FAZ 16.07.2003). Um eine Fortführung der Regionalpark-Projekte sicherzustellen, wurden Mittel der EU bzw. des Landes Hessen beantragt, deren Kofinanzierung der Planungsverband übernommen hat, obwohl sich diese Aufgabe außerhalb seines Zuständigkeitsbereichs befindet (PVFRM 23.04.2003). Die Widerstände, die eine erfolgreiche Regionalisierung des Regionalparks bislang verhindert haben, sind dabei jedoch nicht nur politischer Art: So verweigert bspw. die Stadt Eschborn einen Beitrag zur lokalen Regionalpark$\mathrm{GmbH}$, weil sie von dem Projekt nicht ausreichend profitieren würde, während andernorts Landwirte den Verlust an landwirtschaftlicher Nutzfläche durch den Regionalpark beklagen (vgl. FAZ 10.06.2003).

Die Handlungsunfähigkeit der politischen Akteure bzw. die Tatsache, dass die Politik für die Struktur der Region Rhein-Main nur unzureichende Lösungen gefunden hat, führte dazu, dass von anderer Seite Vorschläge für eine Regionalreform vorgelegt wurden. Im Folgenden sollen daher die "Metropolitana" und die Bewerbung der Region um die Ausrichtung der Olympischen Sommerspiele 2012 als Beispiele anderer Regionalisierungen erläutert werden. 


\subsection{Die „Regionale“ bzw. „Metropolitana“}

Noch während der Beratungen zum Ballungsraumgesetz begann mit einer Serie in der Frankfurter Rundschau eine neue Regionalinitiative. Ziel sollte eine „Landschafts- und Strukturausstellung" nach dem Vorbild der „IBA Emscher Park“ sein. Die Idee war 1999 im Rahmen einer Veranstaltung der "Wirtschaftsinitiative Frankfurt Rhein-Main" 2 vorgestellt worden. Während des Jahres 2000 wurden in einer Serie der Frankfurter Rundschau Akteure aus Wirtschaft, Kultur und Politik zu der vorliegenden Idee interviewt (Frankfurter Rundschau 2001). Alle befragten Akteure waren sich einig, dass die Region Rhein-Main einer Klammer bedürfe, die der Entwicklung einer Identität der Region förderlich sei, und die Positionierung bzw. das Marketing der Region erleichtern würde. Das Projekt lief dabei unter dem Arbeitstitel „Regionale“. Allen Artikeln der Serie der Frankfurter Rundschau ist gemein, dass die Region Rhein-Main im Mittelpunkt des Interesses steht, ohne dass eine Konkretisierung dieser Region vorgenommen worden wäre. Die Verbindung der unterschiedlichen Akteure war - als kleinster gemeinsamer Nenner - ein gemeinsames Interesse an der Region. Die Tatsache, dass hier eine Region "gemacht“ wurde, ohne auf konkrete Grenzen zu verweisen, kann als eine Regionalisierung im Sinne Werlens (s.o.) verstanden werden, deren Ziel die Erhaltung des Rhein-Main-Gebietes als herausragender Wirtschaftsstandort ist.

Zur Realisierung dieses Ziels wurde - finanziert durch Unternehmen der Region --von einer Arbeitsgruppe begonnen, im Rahmen einer "Machbarkeitsstudie“ (vgl. Lutzky/Fischer 2002) Projektvorschläge zu sammeln, die von der nun als "Metropolitana“ bezeichneten Initiative realisiert werden sollten. Getragen werden sollte die „Metropolitana“ - die auch dazu dienen sollte, „FrankfurtRheinMain“ als „europäische Modellregion" des "corporate citizenship" zu profilieren (Lutzky/Fischer 2002, S. 9) - von regionalen Unternehmen. Von den rund 60 Projektvorschlägen wurden allerdings nur wenige als Pilotprojekte umgesetzt.

Im Lauf des Jahres 2002 zeigte sich jedoch, dass das Potenzial für zwei von der Wirtschaft getragene Regionalinitiativen nicht ausreicht: Aus der Konkurrenz zwischen der „Wirtschaftsinitiative Frankfurt RheinMain“ und der „Metropolitana“ ging die Wirtschaftsinitiative letztlich als Sieger hervor: Im Februar 2003 wurden beide Vereinigungen verschmolzen zur "Wirtschaftsinitiative Metropolitana FrankfurtRheinMain". Was von der "Metropolitana" übrig blieb, waren der Name und eine Idee - das Engagement zur Förderung eines gemeinsamen Image der Region Rhein-Main wurde von der Wirtschaftsinitiative aufgegriffen und weiter verfolgt, u.a. durch ein eigenes Forschungspro- jekt zur Geschichte der Region (vgl. WOLF et al. 2002). Auch hier ist der Versuch erkennbar, auf die politischen Entscheider einzuwirken und eine den Interessen der Mitglieder entsprechende Regionalstruktur zu empfehlen. Die Tatsache, dass sich Unternehmen für eine Regionalreform einsetzen, erinnert im Übrigen an den Verband Region Stuttgart, vor dessen Gründung die Unternehmen der Region in einer Umfrage der IHK betont hatten, dass eine neue Regionalstruktur gleichzeitig auch regionale Handlungsfähigkeit bedeute und ein positiver Standortfaktor der Region sei (Philippi 1995, S. 34).

Mit der „Fusion" von Wirtschaftsinitiative und „Metropolitana" fand außerdem noch eine andere, interessante Entwicklung statt: Während zuvor nur die Frankfurter Rundschau über die „Metropolitana“ berichtet hatte, wurde die Regionalreform nun zu einem Topthema der Frankfurter Allgemeinen Zeitung (vgl. FAZ 17.02.2003, 22.02.2003, 08.03.2003a/b), die sich in regionalpolitischen Berichterstattung zuvor eher zurückhielt - auch dies ein Zeichen dafür, dass die Debatte breitere Gesellschaftsschichten erreichte. In allen Zeitungsartikeln wurde jedoch deutlich, dass sich der wichtigste Akteur einer neuerlichen Reform verweigert - der hessische Ministerpräsident Koch sagte immer wieder, dass er keinen aktuellen Handlungsbedarf sehe, sondern abwarten wolle, wie sich die mit dem Ballungsraumgesetz entworfene Struktur entwickle. So sagte ein Regierungssprecher in Bezug auf die freiwilligen Kooperationen: „Der Gärungsprozess in der Region ist noch nicht zu Ende gekommen. Diesen Prozess wollen wir nicht stören" (FAZ 17.02.2003). Dabei zeigen gerade andere Beispiele - z.B. die Regionen Hannover, Stuttgart oder London (Priebs 1999, S. 26-27; Phillipi 1995, 35; Thornley 2002, S. 49) -, dass nur dann Regionen entstehen, wenn dies von einer übergeordneten politischen Ebene durchgesetzt werden. So war die Bildung der Regionen Hannover und Stuttgart, die in Deutschland noch immer als beispielgebend gelten, nur durch Initiativen der Landesregierungen möglich. In London war es die 1997 gewählte Regierung unter Blair, die 1999 mit der Greater London Authority eine demokratisch verfasste und auf Entwicklungsstrategien ausgerichtete Regionalstruktur schuf (vgl. Thornley 2002, S. 49). Insofern hat die hessische Landesregierung recht getan, sich dem Thema „Regionalreform“ $\mathrm{zu}$ widmen. Wenig tragfähig war jedoch der Ansatz, die Kooperation in der Region fast ausschließlich auf Freiwilligkeit aufzubauen. Steinacher meint dazu, dass „...eine echte solidarische Gesamtleistung für eine Region ... auf freiwilliger Basis nicht zu schaffen [ist]" und plädiert für umfassende Regionalverbände nach dem Vorbild der Region Stuttgart (Steinacher 1998, S. 96). Diese "Zerbrechlichkeit" freiwilliger Kooperationen 
lässt sich auch im Rhein-Main-Gebiet an Beispielen nachvollziehen: So führte der angekündigte Umzug eines Madonnen-Gemäldes von Hans Holbein d.J. aus dem Hessischen Landesmuseum in Darmstadt in das Frankfurter Städel-Museum zu Auseinandersetzungen innerhalb der „Kulturinitiative Rhein-Main“, die in der Drohung der Stadt Darmstadt gipfelten, die Initiative $\mathrm{zu}$ verlassen (vgl. FAZ 14.08.2003a und b, FR 29.07.2003, 15.08.2003).

Die Tatsache, dass sich die Landesregierung einer umfassenden Regionalreform bislang verweigert hat, obwohl diese Forderung auch von anderen regionalen Akteuren aufgegriffen worden ist und von den größten regionalen Unternehmen getragen wird, unterstreicht, wie wenig die Regierung im Regionalisierungsprozess auf Einwände anderer Akteure achtet und geachtet hat. Freund (2002, S. 143) verkennt daher die Lage, wenn er in Hinblick auf die „Metropolitana“ äußert, dass „,economic agents have remained leaders in metropolitan issues". In der Region Stuttgart hat die Forderung der Unternehmen nach einer handlungsfähigen Region als Standortfaktor unter anderem zur Schaffung der aktuellen Regionalstruktur geführt. Möglicherweise war jedoch Ausschlag gebend, dass der Raum Stuttgart stärker von produzierenden Unternehmen geprägt und somit strukturell anfälliger gegenüber Krisen ist. Dem zu Folge wäre der Leidensdruck, eine „echte“ Regionalreform im Rhein-Main-Gebiet umzusetzen, noch nicht groß genug. Gleichwohl deuten sich auch im RheinMain-Gebiet Strukturveränderungen wichtiger Branchen an, so dass man folgern könnte, dass auch im Rhein-Main-Gebiet eine handlungsfähige Regionalstruktur als positiver Standortfaktor gelten könnte.

\subsection{Die Bewerbung für die Olympischen Sommerspiele 2012}

Bereits in den 1980er Jahren gab es im Rhein-MainGebiet Überlegungen, sich als Austragungsort der Olympischen Spiele 2004 zu bewerben. Allerdings hatte Frankfurt seine Bestrebungen seinerzeit zu Gunsten Berlins aufgegeben. In Erinnerung geblieben ist den Akteuren jedoch, dass die Olympia-Planungen bereits damals eine Aufbruchstimmung in der Region initiiert hatten (FR 01.03.2001a).

Den erneuten Anstoß, an einer Bewerbung um die Ausrichtung der Olympischen Spiele zu arbeiten, gab der hessische Innenminister Bouffier mit einem Schreiben an die Frankfurter Oberbürgermeisterin Roth 2001. Die Idee stieß auf offene Ohren, z. B. bei zahlreichen Oberbürgermeistern sowie dem Landrat des Main-KinzigKreises (FR 01.03.2001b). Zeitgleich erfolgte die Gründung einer „Olympiainitiative Frankfurt Rhein-Main“ mit Unterstützung des Ministerpräsidenten Koch (FR 02.03.2001). Zudem konnte die Olympia-Initiative auf eine breitere Basis gestellt werden, da der Direktor des Umlandverbandes Frankfurt FAUST ankündigte, dass sich der ab 01.04.2001 aktive Planungsverband des Themas annehmen werde (FR 28.03.2001).

Auf der Regionalkonferenz im Mai 2001 wurde entschieden, dass die Region sich beim Nationalen Olympischen Komitee (NOK) darum bewerben solle, als Kandidat an der internationalen Ausscheidung teilnehmen zu dürfen. Formal sollte sich die Stadt Frankfurt bewerben, um den Regeln des Internationalen Olympischen Komitees (IOC) zu genügen (FR 12.05.2001). Auch die „Wirtschaftsinitiative Frankfurt Rhein-Main“ legte am 08.05.2001 eine positive Stellungnahme zur Olympiabewerbung vor. Mit dem „Rat der Region“ hatten sich dann alle existierenden regionalen Gremien für eine Olympiabewerbung der Region ausgesprochen (FR 27.06.2001, FAZ 27.06.2001). Allein die Tatsache, einen regionalen Konsens in dieser Breite gefunden zu haben, war für die Region RheinMain ein großer Fortschritt.

Die Allianz zur Olympiabewerbung erhielt weiteren Auftrieb mit einer Machbarkeitsstudie (Oktober 2001), die die Austragungsorte in der Region und die Planungen zum Olympiastadion sowie dem Olympischen Dorf in Frankfurt vorstellte (vgl. FAZ 24.10.2001, FR 24.10.2001). Auch der hessische Landtag stellte sich hinter die Bewerbung (FR 26.10.2001).

Während Politiker aller Parteien und Ebenen hinter der Bewerbung standen, gab es auch kritische Stimmen aus der Region. Hier sei z.B. die Frankfurter Sportdezernentin Schenk genannt, die der Ansicht war, die Region habe mit ihrer Bewerbung keine Chance auf die Olympiade. Auch das in der Machbarkeitsstudie enthaltene Konzept für Sportstätten und Olympisches Dorf rief Unmut hervor, lag doch das geplante Olympische Dorf teilweise in einer Kleingartenanlage, so dass die Kleingartenbesitzer protestierten (FR 27.10.2001).

Für die Koordination der Bewerbung wurde noch 2001 eine „Frankfurt Rhein-Main GmbH“ gegründet, deren Gesellschafter die Stadt Frankfurt, das Land Hessen, die Wirtschaftsinitiative Frankfurt Rhein-Main und der Planungsverband waren. Die Landkreise entschieden sich schnell, die Bewerbung ebenfalls finanziell mit zu unterstützen (vgl. FR 13.12.2001), so dass die Gesellschaft ihre Bewerbung Mitte 2002 an das NOK übergab (FAZ 16.05.2002).

Bis zur Präsentation der Olympia-Bewerbung am 12.04.2003 wurde die Bewegung von einem breiten regionalen Konsens getragen. Mit Werbeaktionen wurde versucht, ehrenamtliche Helfer zur Unterstützung 
der Durchführung der Olympischen Spiele anzuwerben und die Bewerbung den Menschen der Region nahe zu bringen. Insbesondere kurz vor der Entscheidung des NOK waren überall in der Region Plakate mit Hinweisen auf die Bewerbung zu sehen. Dennoch hatte das Engagement der Region letztlich nicht den gewünschten Erfolg, denn Frankfurt schied relativ früh im Auswahlverfahren aus.

Die Tatsache, dass Akteure aus Wirtschaft und Politik das Projekt „Olympia-Bewerbung“ gemeinsam initiiert und getragen haben, zeigt dass im Rhein-Main-Gebiet das Potenzial für regionale Kooperation vorhanden ist. Im konkreten Fall reichte es sogar so weit, dass sich die öffentlichen Gesellschafter der "Frankfurt Rhein-Main $2012 \mathrm{GmbH}^{\prime \prime} \mathrm{zu}$ einem finanziellen Engagement entschlossen. Der Gedanke, der dabei alle einte, war die Idee, gemeinsam eine Großveranstaltung in die Region zu holen, von der alle Akteure profitiert hätten. Insofern ist auch die Olympiabewerbung als ein zielgerichteter Zusammenschluss regionaler Akteure eine Regionalisierung, die man unter die Überschrift "Olympiaregion“ stellen könnte. Man darf jedoch nicht übersehen, dass die Olympiabewerbung eine Sondersituation war: Genauso wie sie zu einer WinWin-Situation für alle Beteiligten hätte werden können, genau so weit ist eine Olympiade in der Regel vom politischen Alltagsgeschäft entfernt. Dies mag mit einer der Gründe sein, warum die erfolgreiche Kooperation im - unverfänglichen - olympischen Terrain bislang keine Chance auf Übertragbarkeit in andere, politisch sensiblere Bereiche hatte.

\section{Schluss}

In Bezug auf eine umfassende Regionalreform im Rhein-Main-Gebiet wird immer wieder deutlich, dass das Zusammenwirken der Akteure aus Wirtschaft, Lokal- und Regionalpolitik, die Erarbeitung neuer Vorschläge zur Regionalstruktur oder auch die Kritik am bestehenden Ballungsraumgesetz nur Erfolg versprechen, wenn die Landesregierung willens ist, auf die vorgebrachte Kritik zu reagieren. Die Unzufriedenheit der Kommunen, die zur Mitgliedschaft im „Planungsverband Ballungsraum Frankfurt/Rhein-Main“ verpflichtet sind, hat sich u.a. in einer Klage vor dem hessischen Staatsgerichtshof geäußert, da sich einige Kommunen durch das Gesetz in ihrer Selbstverwaltungshoheit eingeschränkt sehen. Die Klage wiederum wird von der Landesregierung verwendet, um weitere Entscheidungen zu vertagen, bis das Verfahren abgeschlossen ist, wobei die Klage gleichzeitig eine Rechtsunsicherheit in der Region geschaffen hat, die die Handlungsgrundlage der verschiedenen Elemente des Ballungsraumgesetzes einschränkt.
Das Ballungsraumgesetz bietet an sich einen interessanten Alternativansatz zu anderen verfassten Regionen, die es bislang in Deutschland gibt - wenn auch einige Elemente fehlen (z.B. die direkte demokratische Legitimation), so könnten die vorhandenen Elemente doch besser genutzt werden, z.B. durch den Ausbau der Koordinationsfunktion des "Rates der Region“.

Insgesamt zeigt der Blick auf die aktuellen Regionalisierungsprozesse in der Region Rhein-Main, dass die Landesregierung die einzige Instanz ist, die eine handlungsfähige Region schaffen könnte. Dabei ist zu beobachten, dass es für Akteure außerhalb der Politik schwer ist, eigene Ideen in den Entscheidungsprozess zu integrieren. Demnach hat Bahrenberg (2002, S. 63) Recht, wenn er bemerkt, dass keine Regionalisierung ohne die Politik möglich wäre. Allerdings müsste gerade beim Fallbeispiel Rhein-Main gefragt werden, inwiefern es seitens der politischen Akteure erwünscht ist, andere mit ihren Ideen und Vorschlägen in die politischen Regionalisierungsprozesse $\mathrm{zu}$ integrieren. Und: Es ist ein Trugschluss anzunehmen, dass die Politik sich selbst darum bemüht, externe (z.B. wissenschaftliche) Regionalisierungen in diesen Prozessen zu berücksichtigen. Für die Region Rhein-Main kann man feststellen, dass versucht wurde, wissenschaftliche Regionalisierungen in die politischen Entscheidungsprozesse zu tragen (z.B. Wolf/Scheller 2001). Jedoch wurden diese von der Politik nicht wahrgenommen; die Politik zeigt sich hier „beratungsresistent“. So wie die Aufstellung des Ballungsraumgesetzes durchgeführt wurde, hat es den Anschein, als hätten hier eher persönliche Absichten der politisch Verantwortlichen den Ausschlag gegeben und weniger die Inhalte der gemachten Vorschläge. Dies unterstreicht noch einmal, dass es sinnvoll ist, sich Regionalisierungsprozessen mit einem handlungstheoretischen Ansatz zu nähern, denn schließlich sind dieselben Akteure, die für Regionalisierungsprozesse verantwortlich sind, auch für andere Gesetze verantwortlich, die gesellschaftliche Institutionen sowie Regeln gesellschaftlichen Handelns bestimmen.

Insgesamt hat die Landesregierung durch das Ballungsraumgesetz eine Handlungseinschränkung geschaffen, die die Umsetzung anderer Regionalisierungen - initiiert von anderen Akteuren - wirksam behindert. Das Ballungsraumgesetz ist mit seinem Konzept als eine politische Regionalisierung bzw. Territorialisierung zu begreifen, die versucht, die hinsichtlich ihrer wirtschaftlichen Stärke innerhalb des Bundeslandes Hessens übermächtige Region Rhein-Main nicht so sehr zu ermächtigen, dass sie ein politisches Gegengewicht zur Landesregierung werden könnte. Nach der oben formulierten Fragestellung, ob die regi- 
onalen Strukturen in der Lage sind, ausreichende Handlungsmöglichkeiten zu eröffnen, so dass sich die Region künftig im Wettbewerb behaupten kann, lässt sich bislang sagen, dass die Handlungsmöglichkeiten der Region derzeit eingeschränkt sind: Dies gilt für die „beratungsresistente“ Landesregierung wie für diejenigen, die die im Ballungsraumgesetz eingeräumten Handlungsmöglichkeiten nicht optimal nutzen. Dass Möglichkeiten zur Kooperation bestehen, zeigt die gemeinsam auf den Weg gebrachte Olympia-Bewerbung, die gleichzeitig ein Beleg für beginnendes „regionales Denken“ ist. Ob dazu z.B. die Regionalkonferenzen beitragen können und so - wie Esser (2003) meint eine "schleichende" Regionalisierung „von unten" befördern, bleibt abzuwarten. Wie jüngst begonnene Debatten und Vorschläge zur Regionalreform aber andeuten, kommt dem Ballungsraumgesetz das „Verdienst“ $\mathrm{zu}$, diese Diskussionen in positiver Weise befördert zu haben - insofern ist das Gesetz, wenn auch indirekt, durchaus ein Erfolg.

\section{Anmerkungen}

(1)

Der "Regionalpark Rhein-Main" dient einerseits der Freiraumsicherung in der Region, soll aber andererseits auch dazu beitragen, die Region durch Kunstwerke, Aussichtstürme und ein Wegenetz (sog. „Regionalparkrouten“) greif- bzw. erlebbarer zu machen. Das Projekt hat neben seiner Bedeutung als Freizeitund Erholungslandschaft auch einen eigenen Stellenwert als weicher Standortfaktor.

(2)

Die „Wirtschaftsinitiative Frankfurt Rhein-Main“ (seit Februar 2003: „Wirtschaftsinitiative Metropolitana Frankfurt RheinMain") ist ein Zusammenschluss von Unternehmen aus der Region mit dem Ziel, „die Region Rhein-Main zu fördern, damit sie im internationalen Wettbewerb der Regionen auch weiterhin eine führende Position einnimmt" (WirtschaftsinitiativeMetropolitana Frankfurt Rhein-Main 2003).

\section{Literatur}

Bahrenberg, G. (2002): Globalisierung und Regionalisierung: die "Enträumlichung" der Region. In: Geographische Zeitschrift, H. 1, S. 52-63

Esser, J. (2003): Politisch-institutionelle Bedingungen der Region Rhein-Main für die Gestaltung der Qualität der Arbeit. - Manuskript zum Vortrag im Rahmen des 3. Wissenschaftsforums des Rhein-Main-Instituts am 3.12.2003 in Darmstadt

$\mathrm{FAZ}=$ Frankfurter Allgemeine Zeitung

FAZ (24.10.2001): Olympiastadion soll auf Frankfurter Rebstockgelände, S. 61
FAZ (16.05.2002): Erreichbarkeit und Internationalität als Trümpfe, S. 53

FAZ (17.02.2003): An einem Strick ziehen die Politiker nur selten., S. 43

FAZ (22.02.2003): Wirtschaftsinitiative fordert Reform der Regionalstruktur, S. 63

FAZ (08.03.2003a): Warten auf Koch (Kommentar), S. 55

FAZ (08.03.2003b): Regionalreform: Regierung wartet Urteil des Staatsgerichtshofs ab,S. 55

FAZ (10.06.2003): Regionalpark-Idee stößt bei vielen Landwirten auf Skepsis. - (www.faz.net)

FAZ (16.07.2003): Dachgesellschaft für Regionalpark. Landkreise gründen neue GmbH,S. 51

FAZ (14.08.2003a): Holbein-Madonna - Darmstadt: Keine Kooperation mehr mit Frankfurt (www.faz.net)

FAZ (14.08.2003b): Maria, hilf! (Kommentar). - (www.faz.net)

\section{FR $=$ FRANKFURTER RUNDSCHAU}

FR (17.08.2000): Herkströter will Direktor werden, S. 33

FR (24.08.2000): Ein Gesetz für die Region, das die Region nicht will, S. 39

FR (2001): Die Zukunft der Regionen. Die Landschafts- und Strukturausstellung "Regionale“. Eine Serie der Frankfurter Rundschau. - Frankfurt

FR (01.03.2001a): Appetithappen,S. 37

FR (01.03.2001b): Große Koalition für die Olympia-Bewerbung, S. 37

FR (12.05.2001): Mit einer „Allianz für Olympia“ tritt Rhein-Main an, S. 33

FR (27.06.2001): Rat der Region will Olympiabewerbung, S. 29

FR (24.10.2001): Bahn-Tochter hält an eigenen Plänen fest, S. 25

FR (26.10.2001): Alle vier Fraktionen sind für Olympia, S. 35

FR (27.10.2001): Kleingärtner gehen gegen Olympia-Planung auf die Barrikaden, S. 25

FR (13.12.2001): 100000 Euro für die Olympia-Bewerbung, S. 1

FR (29.07.2003): Madonnas Umzug. Darmstadts Holbein-Gemälde soll ins Städel kommen. - (www.fr-aktuell.de)

FR (15.08.2003): Madonna hilf! Wie Holbeins Schöne die RheinMain-Region entzweit, S. 23

Freund, B. (2002): The Frankfurt Rhine-Main Region. In: Salet W.; Thornley, A.; KreukelS, A. (2002). -London, S. 125-144 
Krenzlin, A. (1961): Werden und Gefüge des rhein-mainischen Verstädterungsgebietes. - In: Frankfurter Geographische Hefte, H. 37, S. 311-387. -Frankfurt

Lutzky, N.; Fischer, R. (2002): Metropolitana FrankfurtRheinMain. Von der Idee zur Realisierung. Die Wirtschaft als Partner der Bürgergesellschaft. Machbarkeitsstudie. - Frankfurt

Philippi, T. (1995): Interkommunale Zusammenarbeit (II) - Gesetzliche Regelungen für die Region Stuttgart. In: Standort, H. 1, S. 34-36. -Heidelberg

Priebs, A. (1999): Die Region - notwendige Planungs- und Handlungsebene in Verdichtungsräumen. Erfahrungen und Perspektiven im Großraum Hannover. - In: Rhein-Mainische Forschung, H. 116, S. 11-33, Frankfurt

PVFRM (23.04.2003): Ballungsraumgesetz keine regionale Lösung - Planungsverband gut aufgestellt. (Pressemitteilung des Planungsverbands Ballungsraum Frankfurt/Rhein-Main)

Rhein-Mainische Forschung (2000): Regionalatlas Rhein-Main. $=$ Rhein-Mainische Forschungen, H. 120. -Frankfurt

Ruppert, K.; Paesler, R. (1983): Raumorganisation in Bayern. Neue Strukturen durch Verwaltungsgebietsreform und Regionalgliederung. = WGI-Berichte zur Regionalforschung H. 16, München

Schamp, E. (2001): Der Aufstieg von Frankfurt/Rhein-Main zur europäischen Metropolregion. - In: Geographica Helvetica, H. 3, S. $169-178$

Scheller, J. (1998): Rhein-Main. Eine Region auf dem Weg zur politischen Existenz. = Materialien, H. 25, Frankfurt

Scheller, J. (1999): Auf der Suche nach den Grenzen der Region. Die Rolle territorialer Abgrenzungen in der aktuellen rhein-mainischen Regionalreformdebatte. - In: Rhein-Mainische Forschungen, H. 119, S. 313-360, Frankfurt

Schultheis, J. (2002): An der Verteilung des Geldes beißen sich alle die Zähne aus. Hessens kommunaler Finanzausgleich benachteiligt große Städte / Reformen immer wieder gefordert / Regionalkreis-Modell favorisiert. In: Frankfurter Rundschau, 31.10.2002, S. 35, Frankfurt

Schultheis, J. (2003): Zwischen Heimatgefühl und Weltstadtanspruch. Die Region Frankfurt/Rhein-Main. In: DISP, H. 152, S. 80-86, Zürich

Spahn, P.; van den Busch, U. (2002): Position und Entwicklungsperspektiven des Finanzplatzes Frankfurt. = FEH-Report, H. 645, Wiesbaden
Steinacher, B. (1998): Verband Region Stuttgart. - In: EMPIRICA (1998): Die Zukunft der Stadtregionen. Dokumentation eines Kongresses in Hannover am 22. und 23. Oktober 1997. S. 93-96, Bonn

Thornley, A. (2002): London: institutional turbulence but enduring nation-state control. - In: Thorley, A.; Salet, W. und Kreukels, A. (2002): Metropolitan Governance and Spatial Planning. Comparative Case Studies of European City-Regions. S. 41-56, London

Werlen, B. (2001a): Sozialgeographie. Eine Einführung. - Bern, Stuttgart, Wien

Werlen, B. (200lb): Alltägliche Regionalisierungen. - In: Brunotte, E.; Gebhardt, H.; Meurer, M.; Meusburger, P.; Nipper, J. (Hrsg.)(2001): Lexikon der Geographie. Bd. 1, S. 40-42, Heidelberg

Wiechmann, T. (2000): „Die Region ist tot - es lebe die Region!" Animerkungen zur Diskurskonjunktur und Relativierung des Begriffs. In: Raumforschung und Raumordnung, H. 2-3/2000 S. 173-184

Wirtschaftsinitiative Metropolitana Frankfurt Rhein-Main (2003) - Homepage (www.wifrm.de) (18.08.2003)

Wolf K. (1990): Entwicklungstendenzen der Wirtschaft in der Rhein-Main-Region. - In: Rhein-Mainische Forschung, H. 107, S. 25-58, Frankfurt

Wolf, K. (2003): Die Region Rhein-Main im Europa der Regionen. In: Geographie und Schule, H. 144, S. 16-23

Wolf, K.; Langhagen-Rohrbach, C.; Gretschel, S. (2002): Die Region Rhein-Main - Lebensraum und Funktionalregion. - unveröff. Gutachten, Frankfurt

Wolf, K., Scheller, J. (2001): Stellungnahme zum Gesetzentwurf der Landesregierung für ein „Gesetz zur Stärkung der kommunalen Zusammenarbeit und Planung in der Region Rhein-Main (BallungsraumG). $=$ KSR36, H. 2, Frankfurt

Dr. Christian Langhagen-Rohrbach Institut für Kulturgeographie, Stadt- und Regionalforschung der Johann Wolfgang Goethe-Universität Senckenberganlage 36 60325 Frankfurt am Main

E-Mail: Rohrbach@em.uni-frankfurt.de 